\title{
APPENDIX A: EUROPEAN FACILITIES USING NUCLEAR TECHNIQUES TO STUDY CULTURAL HERITAGE
}

The map below shows laboratories and centres with facilites relevant to nuclear physics studies of Cultural Heritage objects. These are grouped into four categories:

$\square$ Ion Beam Analysis Facilities in Europe

$\square$ European Accelerator Mass Spectrometry Facilities

$\square$ European Neutron Sources

$\square$ Other European Centres, Facilities and Laboratories

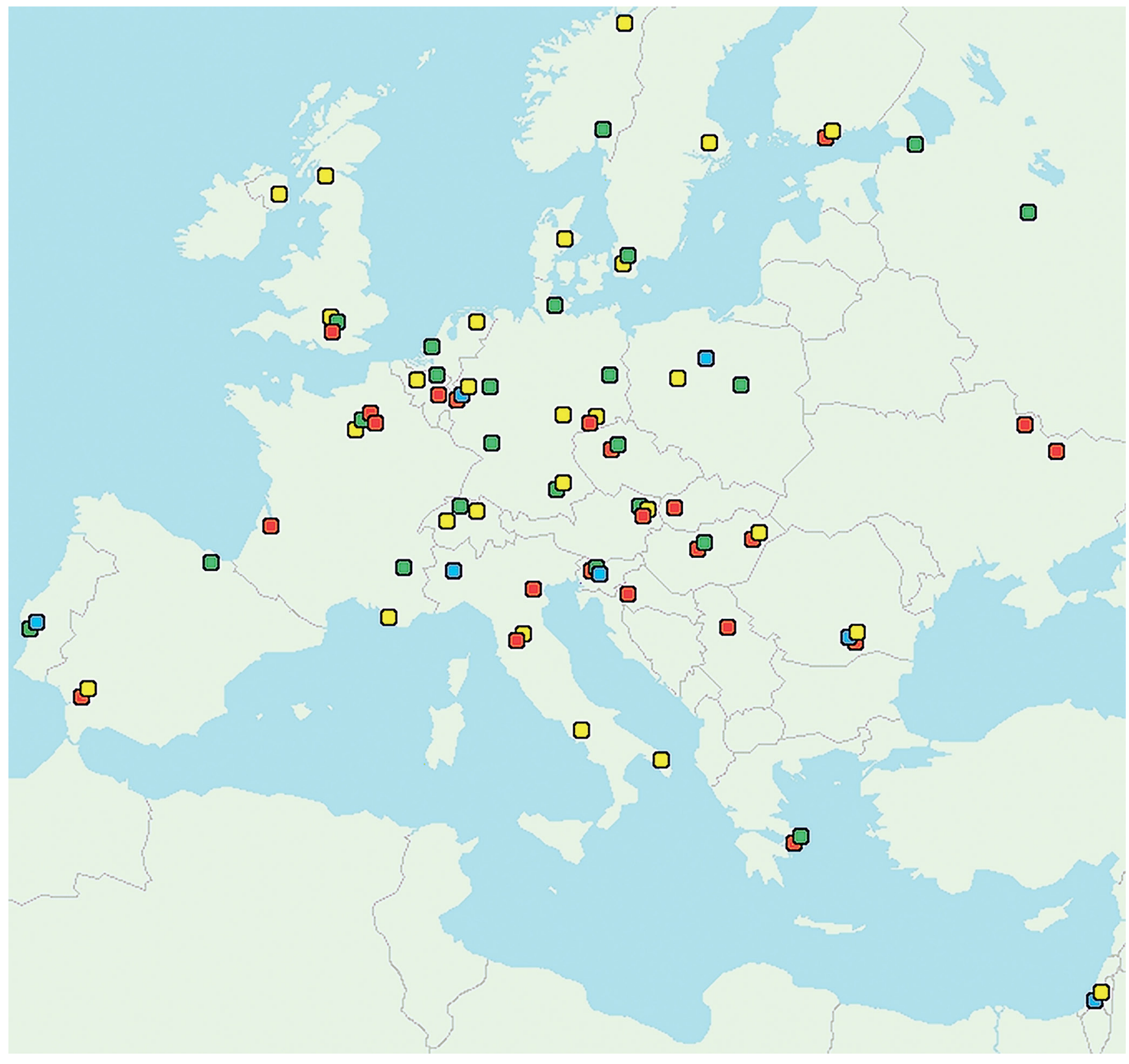




\section{ION BEAM ANALYSIS FACILITIES IN EUROPE}

Austria, NSIL Nuclear Science and Instrumentation Laboratory, IAEA, Seibersdorf, https://www.seibersdorf-laboratories.at/en/home

$\square$ Belgium, Centre Européen d'Archéométrie (CEA) \& Institut de Physique Nucléair, University of Liege, http://www.cearcheo.ulg.ac.be/Accueil.html

$\square$ Belgium, ALTAïS Accélérateur Linéaire Tandetron pour l'Analyse et l'Implantation des Solides, Namur http://www.unamur.be/sciences/physique/larn/

- Croatia, Laboratory for Ion Beam Interactions, Ruđer Bošković Institute, CEMS Centre of Excellence for Advanced Materials and Sensing Devices, Zagreb, http://cems.irb.hr/en/

$\square$ Czech Republic, CANAM Centre for Accelerators and Nuclear Analytical Methods, Nuclear Physics Institute, ASCR Academy of Sciences of the Czech Repullic, Řež, http://canam.ujf.cas.cz/

$\square$ Finland, University of Helsinki, Ion beam analysis laboratory, Helsinki, https://tuhat.halvi.helsinki.fi/portal/en/equipment/ion-beam-analysis-1(2c267889-4d75-446f-95b3-c1384de3e9c4).html

- France, AGLAE, Accélérateur Grand Louvre d'Analyses Elémentaires, Paris http://c2rmf.fr/analyser/un-laboratoire-de-haute-technologie-pour-les-collections-des-musees/aglae

口 France, CENBG Centre d'Études Nucléaires de Bordeaux Gradignan, http://www.cenbg.in2p3.fr/

$\square$ France, SAFIR Système d'Analyse par Faisceaux d'Ions Rapides, Institut des NanoSciences de Paris, http://www.insp.jussieu.fr/Systeme-d-Analyse-par-Faisceaux-d.html

$\square$ Germany, HZDR Helmholtz Zentrum Dresden-Rossendorf, Ion Beam Analysis Group, Dresden, http://www.hzdr.de/db/Cms?pOid=35575\&pNid=2744

$\square$ Greece, NCSR National Centre for Scientific Research DEMOKRITOS, Athens, http://www.demokritos.gr/

$\square$ Italy, INFN LABEC, Florence, http://labec.fi.infn.it/

— Italy, Laboratori Nazionali di Legnaro, AN2000 accelerator, INFN Legnaro, http://www.Inl.infn.it/index.php/en/ accelerators-3/an-2000

- Hungary, MTA Atomki, Laboratory of Ion Beam Applications, Institute for Nuclear Research, Debrecen, http://w3.atomki.hu/index_en.html

$\square$ Hungary, MTA Wigner FK, Department for Materials Science by Nuclear Methods, Wigner Research Centre for Physics, Budapest, http://wigner.mta.hu/en

$\square$ Romania, Horia Hulubei National Institute for Nuclear Physics and Engineering, Bucharest-Magurele, http://www.nipne.ro/

- Serbia, FAMA Facility for Modification and Analysis of Materials with Ion Beams, Vinča Institute of Nuclear Sciences, Belgrade, http://www.vin.bg.ac.rs/010/index.html

$\square$ Slovak Republic, Advanced Technologies Research Institute, Trnava, http://www.mtf.stuba.sk/english/institutes/advanced-technologies-research-institute/

口 Slovenia, MIC Microanalytical Centre, Jožef Stefan Institute (JSI), Ljubljana, http://www.rcp.ijs.si/mic/

$\square$ Spain, Centro Nacional de Aceleradores, CNA, is a joint centre of Universidad de Sevilla, Junta de Andalucía and CSIC, Seville, http://acdc.sav.us.es/cna/

口 United Kingdom, Ion Beam Centre, University of Surrey, Guildford, http://www.surrey.ac.uk/ati/ibc/

$\square$ Ukraine, ISSPMT Institute of Solid State Physics, Materials Science and Technologies, National Academy of Sciences of Ukraine, Kharkov, http://www.kipt.kharkov.ua/kipt_sites/isspmst/main_site/ENG34Main.html

- Ukraine, Institute of Applied Physics, National Academy of Sciences of Ukraine, SUMY, http://iap.sumy.org/viewexpbase/en/?id=15

\section{EUROPEAN NEUTRON SOURCES}

Austria, TRIGA Mark II Reactor, TU Vienna, Vienna, http://ati.tuwien.ac.at/reactor/EN/

$\square$ Belgium, IRMM Institute for Reference Materials and Measurements, Geel, https://ec.europa.eu/jrc/en/institutes/irmm

$\square$ Czech Republic, Department of Neutron Physics, Nuclear Physics Institute, ASCR, Academy of Sciences of the Czech Republic, Řež, http://neutron.ujf.cas.cz/

$\square$ Europe, Central European Research Infrastructure Consortium (CERIC-ERIC), http://www.ceric-eric.eu/

$\square$ France, Institut Laue-Langevin (ILL), Neutrons for Science, https://www.ill.eu/

- France, Laboratoire Léon Brillouin (LLB), Gif-sur-Yvette, http://www-llb.cea.fr/en/

$\square$ Germany, Forschungs-Neutronenquelle Heinz Maier-Leibnitz (FRM II), Garching, https://www.frm2.tum.de/en/home/

$\square$ Germany, GEMS German Engineering Materials Science Centre, Helmholtz-Zentrum Geesthacht, http://www.hzg.de/index.php.de 
Germany, Helmholtz-Zentrum Berlin für Materialien und Energie (HZB), Berlin, https://www.helmholtz-berlin.de/index_en.html

口 Germany, Jülich Centre for Neutron Science, Jülich, http://www.fz-juelich.de/jcns/EN/Home/home_node.html

$\square$ Germany, TRIGA Mark II reactor, Johannes Gutenberg University, Mainz, http://www.kernchemie.uni-mainz.de/eng/234.php

$\square$ Greece, NCSR National Centre for Scientific Research DEMOKRITOS, http://www.demokritos.gr/

- Hungary, Budapest Neutron Centre (BNC), Budapest, http://www.bnc.hu/

T The Netherlands, RID Reactor Institute Delft, Delft, http://www.rid.tudelft.nl/en

$\square$ Norway, IFE Institute for Energy Technology, Kjeller, http://www.ife.no/en

$\square$ Poland, MARIA National Centre for Nuclear Research, Otwock-Świerk, http://www.ncbj.gov.pl/en/maria

口 Portugal, Centro de Ciências e Tecnologias Nucleares (C2TN), Instituto Superior Técnico (IST), Lisbon, http://c2tn.tecnico.ulisboa.pt/en

Russia, Joint Institute for Nuclear Research (JINR), Dubna, http://wwwold.jinr.ru/

口 Russia, PNPI Petersburg Nuclear Physics Institute, Gatchina, St. Petersburg, http://www.pnpi.spb.ru/

$\square$ Slovenia, TRIGA Reactor Infrastructure Centre, Ljubljana, http://www.rcp.ijs.si/ric/reactor-a.htm

$\square$ Spain, ESS Bilbao, Bilbao http://www.essbilbao.org/index.php/en/

$\square$ Sweden, MAX IV and European Spallation Source (ESS), Lund, http://www.lunduniversity.lu.se/research/max-iv-and-ess

$\square$ Switzerland, SINQ: The Swiss Spallation Neutron Source, Paul Scherrer Institute, Villigen, https://www.psi.ch/sinq/

U United Kingdom, ISIS Neutron and Muon Source, Didcot, http://www.isis.stfc.ac.uk/

\section{EUROPEAN ACCELERATOR MASS SPECTROMETRY FACILITIES}

Austria, VERA Vienna Environmental Research Accelerator, Universität Wien, Vienna, https://isotopenforschung.univie.ac.at/en/vera/

$\square$ Belgium, Radiocarbon Dating Laboratory, RICH Royal Institute for Cultural Heritage, Brussels, http://www.kikirpa.be/EN/52/353/Datation+C14.htm

$\square$ Denmark AMS Department. of Physics and Astronomy, Aarhus University, Aarhus, http://phys.au.dk/en/research/research-areas/accelerator-mass-spectroscopy/

$\square$ Finland lon beam analysis laboratory, Department of Physics, University of Helsinki, Helsinki, http://www.physics.helsinki.fi/tutkimus/mat/english/research/ionbeam

- France, ARTEMIS, Pelletron CEA, Saclay, Gif-sur-Yvette, http://www2.cnrs.fr/en/291.htm

$\square$ France, CEREGE - Centre Européen de Recherche et d'Enseignement en Géosciences de l'Environment, Marseille, https://www.cerege.fr/

$\square$ Germany, Max Planck Institute for Biogeochemistry, University of Jena, Jena, http://www.bgc-jena.mpg.de/

$\square$ Germany, GAMS Accelerator Mass Spectrometry Group, Maier-Leibnitz-Laboratory, Garching, http://www.gams.ph.tum.de/

$\square$ Germany, HZDR Helmholtz Zentrum Dresden Rossendorf, Ion Beam Centre, Dresden, http://www.hzdr.de/db/Cms?pOid=27781\&pNid=2791

$\square$ Germany, Centre for Accelerator Mass Spectrometry, University of Cologne, Cologne, http://www.cologneams.uni-koeln.de

$\square$ Hungary, Hertelendi Laboratory of Environmental Studies, Debrecen, http://w3.atomki.hu/hekal/

$\square$ Israel, Max Planck Weizmann Joint Center in the Field of Integrative Archaeology and Anthropology, Weizmann Institute, Rehovot, http://www.weizmann.ac.il/pages/center-of-integrative-archaeology-and-anthropology

$\square$ Italy, CEDAD Centro di Datazione e Diagnostica, Lecce Tandetron Laboratory, Lecce, http://cedad.unisalento.it/en/tandetron.php

$\square$ Italy, CIRCE Center for Isotopic Research on the Cultural and Environmental heritage, seconda universita degli studi di Napoli, Naples, http://www.matfis.unina2.it/dipartimento-205/laboratori/CIRCE

$\square$ Italy, INFN LABEC, Florence http://labec.fi.infn.it/

$\square$ The Netherlands, Radio Carbon AMS, University of Groningen, Groningen, http://www.rug.nl/research/isotope-research/projects/radiocarbon/radiocarbonams

$\square$ Norway, NTNU The National Laboratory of Age Determination, Trondheim, https://www.ntnu.edu/museum/archaeometry

$\square$ Poland, Poznan Radiocarbon Laboratory, Poznan, http://radiocarbon.pl/

$\square$ Romania, Horia Hulubei National Institute for Nuclear Physics and Engineering, Bucharest-Magurele, http://www.nipne.ro/facilities/facilities/tandetron1mv.php 
Spain, Centro Nacional de Aceleradores, CNA, is a joint centre of Universidad de Sevilla, Junta de Andalucía and CSIC, Seville, http://acdc.sav.us.es/cna/

$\square$ Sweden, Radiocarbon Dating Laboratory, Lund University, Lund, http://www.geol.lu.se/c14/en/

$\square$ Sweden, AMS Department of Physics and Astronomy, University of Uppsala, Uppsala, http://www.physics.uu.se/research/applied-nuclear-physics/groups/ion-physics

$\square$ Switzerland, LARA AMS Laboratory, University of Bern, Bern, http://www.14c.unibe.ch/

$\square$ Switzerland, LIP Laboratory for Ion Beam Physics, EHT Zürich, http://www.ipp.phys.ethz.ch/research/ion-beam-physics.html

$\square$ United Kingdom, 14Chrono Centre for Climate, the Environment, and Chronology, Queen's University, Belfast http://www.chrono.qub.ac.uk/

$\square$ United Kingdom, Oxford Radiocarbon Accelerator Unit, Oxford, https://c14.arch.ox.ac.uk/embed.php?File=oxcal.html

$\square$ United Kingdom, SUERC Scottish Universities Environmental Research Centre, East Kilbride, http://www.gla.ac.uk/suerc/

\section{OTHER EUROPEAN CENTRES, FACILITIES AND LABORATORIES}

\section{Magnetic Resonance Centres}

$\square$ Germany, MARC Magnetic Resonance Center, RWTH Aachen University, Aachen www.marc.rwth-aachen.de

$\square$ Slovenia, NMR Centre, Ljubliana http://www.nmr.ki.si/

\section{Gamma Sources}

$\square$ Romania, Extreme Light Infrastructure - Nuclear Physics (ELI-NP), Horia Hulubei National Institute for R\&D in Physics and Nuclear Engineering, Bucharest-Magurele http://www.eli-np.ro/

\section{Luminescence Research}

$\square$ Israel, Geological Survey of Israel, Jerusalem, Luminescence Dating Laboratory, http://www.gsi.gov.il/

$\square$ Poland, Institute of Physics, Faculty of Physics, Astronomy and Informatics, Nicolaus Copernicus University, Toruń, https://www.umk.pl/en/

$\square$ Portugal, Centro de Ciências e Tecnologias Nucleares (C2TN), Instituto Superior Técnico (IST), Lisbon, http://c2tn.tecnico.ulisboa.pt/en

\section{Surface Research}

$\square$ Italy, NIS centre for nanostructured interfaces and surfaces, Universita degli Studi di Torino http://www.nis.unito.it/index.html

\section{X-ray applications}

$\square$ Romania, Accent Pro 2000 S.R.L. (AP2K) Research \& Development Company http://www.accent.ro/

\section{European Research Consortia}

$\square$ Integrated Platform for the European Research Infrastructure ON Culture Heritage (IPERION CH), http://www.iperionch.eu/

$\square$ Central European Research Infrastructure Consortium (CERIC), http://www.ceric-eric.eu/

$\square$ Neutron Scattering and Muon Spectroscopy (NMI3), http://nmi3.eu/

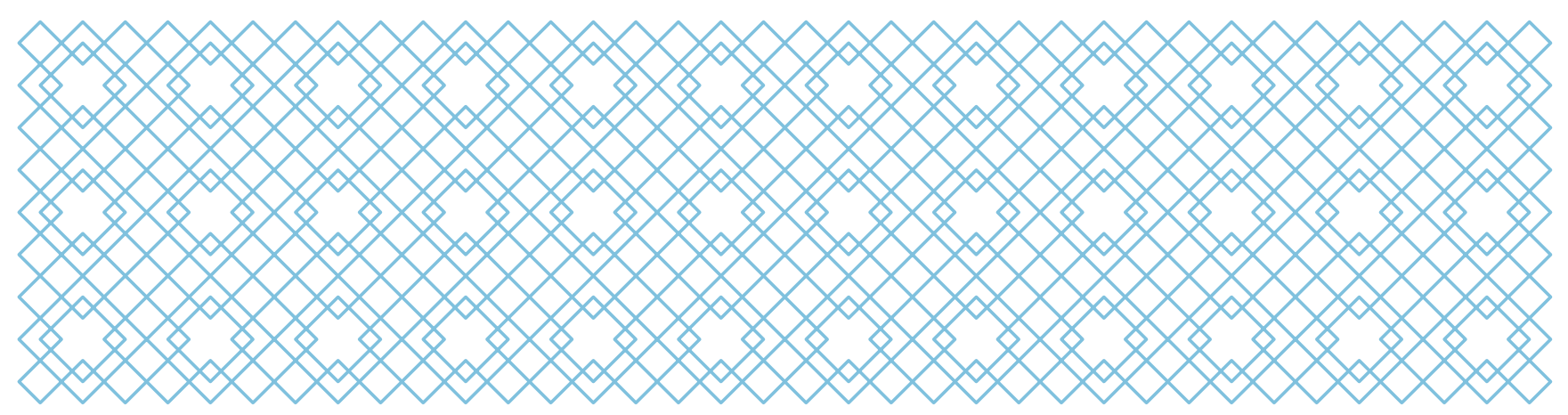

